\title{
More fine structural global square sequences
}

\author{
Martin Zeman
}

Received: 20 February 2009 / Published online: 13 October 2009

(C) The Author(s) 2009. This article is published with open access at Springerlink.com

\begin{abstract}
We extend the construction of a global square sequence in extender models from Zeman [8] to a construction of coherent non-threadable sequences and give a characterization of stationary reflection at inaccessibles similar to Jensen's characterization in $\mathbf{L}$.
\end{abstract}

Keywords Global quare sequence - Fine structure - Extender model ·

Weakly compact cardinal $\cdot$ Stationary reflection

\section{AMS Subject Classification $\quad 03 \mathrm{E} 05 \cdot 03 \mathrm{E} 45 \cdot 03 \mathrm{E} 55$}

This note presents a fine structural construction of a so-called $\square(\kappa, A)$ sequence for certain stationary subsets $A$ of an inaccessible cardinal $\kappa$ as well as a characterization of weakly compact cardinals in fine structural extender models in terms of stationary reflection. These results extend analogous results of Jensen for the constructible universe that originate in [3] and are described in more detail in [1]. Although the characterization of weakly compact cardinals in an extender model turns out to be exactly the same as in $\mathbf{L}$, the proof requires a significant amount of extra work. Also, the author believes that the proof presented in this paper is more straightforward than that described in [3] and [1].

The exposition in this paper is based on extender models with Jensen's $\lambda$-indexing of extenders introduced in [4]; see [7] as a reference. The paper builds on previous work on fine structural square sequences in extender models, in particular on $[5,6]$ and

Research partially supported by NSF grant DMS-0500799.

M. Zeman ( $\square)$

Department of Mathematics, University of California at Irvine, Irvine, CA 92697, USA

e-mail: mzeman@math.uci.edu 
[8]. We will frequently refer to [8]. However, no detailed knowledge of arguments in [8] is necessary, as we will only need certain lemmata from that paper which can be used as black boxes. In particular, all references concerning protomice will be hidden in black boxes.

Theorem 0.1 (Main Theorem) Working in a fine-structural Jensen-style extender model $\mathbf{L}[E]$, assume $\kappa$ is an inaccessible cardinal that is not weakly compact and $A \subseteq \kappa$ is stationary. Then there is a stationary $A^{\prime} \subseteq A$ and a sequence $\left\langle C_{\tau} \mid \tau<\kappa\right\rangle$ satisfying the following conditions.

(a) $C_{\tau}$ is a closed unbounded subset of $\tau$.

(b) $C_{\bar{\tau}}=C_{\tau} \cap \bar{\tau}$ whenever $\bar{\tau} \in \lim \left(C_{\tau}\right)$.

(c) $A^{\prime} \cap \lim \left(C_{\tau}\right)=\varnothing$.

A sequence satisfying (a)-(c) in the above Theorem is called a $\square\left(\kappa, A^{\prime}\right)$-sequence. Any such sequence is a $\square(\kappa)$-sequence, that is, it cannot be threaded: if $C \subseteq \kappa$ is a closed unbounded set then $C \cap \alpha \neq C_{\alpha}$ for some limit point $\alpha$ of $C$. From the above Theorem we obtain the following corollaries, the first of which is immediate.

Corollary 0.2 Let $\mathbf{L}[E]$ be a Jensen-style extender model. The following dichotomy is true in $\mathbf{L}[E]$ of any inaccessible cardinal $\kappa$.

- $\quad \kappa$ is weakly compact $\Longrightarrow$ every stationary subset of $\kappa$ reflects at some $\bar{\kappa}<\kappa$.

- $\quad \kappa$ is not weakly compact $\Longrightarrow$ nonreflecting stationary subsets of $\kappa$ are dense.

Here "dense" means that every stationary subset of $\kappa$ contains a nonreflecting stationary subset.

In particular, an inaccessible cardinal $\kappa$ is weakly compact just in case that every stationary subset of $\kappa$ reflects at some $\bar{\kappa}<\kappa$.

The first clause in the above corollary is, of course, a ZFC consequence. It should be noted that reflection points of stationary subsets whose existence is guaranteed by weak compactness are regular. Not only the argument that is used to obtain reflection points produces regular (in fact inaccessible) reflection points; the fact that we have a global square sequence on singular cardinals in $\mathbf{L}[E]$ guarantees that reflection points of densely many stationary subsets of an inaccessible cardinal $\kappa$ must be regular, up to non-stationarily many. This can be seen by the standard argument for obtaining non-reflection from squares. Given a stationary $A \subseteq \kappa$ where $\kappa$ is inaccessible, it is sufficient to focus on the case where $A$ consists of singular cardinals, as otherwise one can easily prove in ZFC that every reflection point of $A$ must be regular. Using the Fodor's Theorem we can fix the order types of clubs on the global square sequence on some stationary $A^{\prime} \subseteq A$. Letting $C$ be the set of all limit points of $A^{\prime}$, it is a routine to check that any $\delta \in C$ that is a reflection point of $A^{\prime}$ must be regular. The property that every stationary subset of $\kappa$ reflects at some singular ordinal $\bar{\kappa}<\kappa$ or at an ordinal of fixed uncountable cofinality, if consistent with ZFC, must have high consistency strength; however the exact result here is not known. Even at small regular cardinals, the requirement that every stationary set reflects at some ordinal of small cofinality implies the consistency of measurable cardinals of high Mitchell order; see [2]. 
Corollary 0.3 Let $\mathbf{V}=\mathbf{L}[E]$ be a Jensen-style extender model. Then for any regular cardinal $\kappa$ that is not weakly compact there is a Suslin $\kappa$-tree.

This follows from Jensen's construction of higher Suslin trees in [3]. For successor cardinals $\kappa=\mu^{+}$where $\mu$ is not subcompact one uses $\nabla_{\kappa}(A)$ and $\square(\kappa, A)$ for a suitable stationary $A \subseteq \kappa$; here the $\square(\kappa, A)$-sequence is obtained from a $\square_{\mu}$-sequence whose existence is guaranteed by [6]. If $\mu$ is subcompact then $\mu$ is inaccessible, so $\mathrm{GCH}$ in $\mathbf{L}[E]$ makes it possible to construct a Suslin $\kappa$-tree "naively" by using only a $\nabla_{\kappa}\left(S_{\mu}^{\kappa}\right)$-sequence ${ }^{1}$ to seal off large antichains at limit stages of cofinality $\kappa$ in the construction, and adding all possible branches at limit stages of cofinality smaller than $\kappa$. For inaccessible $\kappa$ one constructs a Suslin $\kappa$-tree using $\nabla_{\kappa}(A)$ and $\square(\kappa, A)$ as above; this time the existence of a $\square(\kappa, A)$-sequence is guaranteed by Theorem 0.1 .

\section{The construction}

We will work in a fixed model $\mathbf{L}[E]$ where $E$ is a Jensen-style extender sequence, that is, an extender sequence with $\lambda$-indexing of extenders. The predicate $E$ is thus also fixed. Throughout the construction we will use the Condensation Lemma for premice; this is Lemma 2.2 in [8] or Lemma 9 in [7]. We will often make use of the following simple consequence of the Condensation Lemma.

Proposition 1.1 Assume that $\sigma: \bar{M} \rightarrow M$ is a $\Sigma_{0}$-preserving embedding where $M$ is an $\mathbf{L}[E]$-level and $\bar{M}=\left\langle J_{\bar{\alpha}}^{\bar{E}}, \bar{E}_{\omega \alpha}\right\rangle$ is an acceptable $J$-structure. Let $\tau=\operatorname{cr}(\sigma)$ and $\delta=\tau^{+\bar{M}}$; here we allow the option that $\delta=\operatorname{ht}(\bar{M})$ if $\tau$ is the largest cardinal in $\bar{M}$. If $\tau$ is a limit cardinal in $\bar{M}$ then $\bar{E} \uparrow \delta=E \uparrow \delta$.

From now on assume that $\kappa$ is an inaccessible cardinal that is not weakly compact. As it is typical with constructions of $\square$-like principles, we begin with identifying canonical structures assigned to ordinals $\tau<\kappa$. As $\kappa$ is not weakly compact, there is a $\kappa$-tree on $\kappa$ without a cofinal branch; we fix the $<_{E}$-least one. Thus

$$
T=\text { the }<_{E} \text {-least } \kappa \text {-tree on } \kappa \text { without a cofinal branch. }
$$

Obviously, $T \in J_{\kappa^{+}}^{E}$ and (1) defines $T$ inside $J_{\kappa^{+}}^{E}$. We will write $T \uparrow \tau$ to denote the restriction of $T$ to $\tau$, that is, $T \uparrow \tau$ is the tree on $\tau$ with tree ordering $<_{T} \cap(\tau \times \tau)$.

Lemma 1.2 There is a closed unbounded set of cardinals $\mathcal{C} \subseteq \kappa$ and a map $\tau \mapsto$ $\delta_{\tau}<\tau^{+}$such that for every $\tau \in \mathcal{C}$ we have

- $\tau$ is the largest cardinal in $J_{\delta_{\tau}}^{E}$ and is inaccessible in $J_{\delta_{\tau}}^{E}$.

- $T \uparrow \tau$ is a $\tau$-tree in $J_{\tau}^{E}$ with no cofinal branch in $J_{\delta_{\tau}}^{E}$.

- $T \uparrow \tau$ is an initial segment of $T$, that is, for all $\xi \in T \uparrow \tau$ and all $\zeta \in T$ we have $\zeta<_{T} \xi \Rightarrow \zeta \in T \uparrow \tau$.

\footnotetext{
${ }^{1} S_{\mu}^{\kappa}=\{\xi<\kappa \mid \operatorname{cf}(\xi)=\mu\}$
} 
Proof Let $\left\langle X_{\xi} \mid \xi<\kappa\right\rangle$ be a continuous chain of elementary substuctures of $J_{\kappa^{+}}^{E}$ such that each $X_{\xi}$ is of size $\tau_{\xi}=X_{\xi} \cap \kappa \in \kappa$. Clearly $T \in X_{\xi}$ for all $\xi<\kappa$, as $T$ is definable in $J_{\kappa^{+}}^{E}$. By Proposition 1.1, each $X_{\xi}$ collapses to some $J_{\delta(\xi)}^{E}$. The fact that $X_{\xi} \prec J_{\kappa^{+}}^{E}$ guarantees that $\tau_{\xi}$ is the largest cardinal in $J_{\delta(\xi)}^{E}$, is inaccessible in $J_{\delta(\xi)}^{E}$ and the tree $T$ collapses to $T \uparrow \tau_{\xi} \in J_{\delta(\xi)}^{E}$ that has no cofinal branch in $J_{\delta(\xi)}^{E}$. So we can let $\mathcal{C}=\left\{\tau_{\xi} \mid \xi<\kappa\right\}$ and $\delta_{\tau_{\xi}}=\delta(\xi)$.

To see that $T\left\lceil\tau_{\xi}\right.$ is an initial segment of $T$ it suffices to show that the $\alpha$-th level of $T \uparrow \tau_{\xi}$ agrees with the $\alpha$-th level of $T$ for all $\alpha<\tau_{\xi}$. Fix such an $\alpha$. By elementarity, there is a bijection $f_{\alpha}: \theta_{\alpha} \rightarrow T_{\alpha}$ in $X_{\xi}$ where $T_{\alpha}$ is the $\alpha$-th level of $T$ and $\theta_{\alpha}$ is its size. Since $T$ is a $\kappa$-tree, $\theta_{\alpha}<\kappa$ so $\theta_{\alpha}<\tau_{\xi}$. Then $T_{\alpha}=\operatorname{rng}\left(f_{\alpha}\right) \subseteq X_{\xi}$, as $\theta_{\alpha} \subseteq X_{\xi}$.

Let $\tau \in \mathcal{C}$. Since $T \uparrow \tau$ is an initial segment of $T$ and $T$ has height $\kappa$, the tree $T \nmid \tau$ has a cofinal branch in $\mathbf{L}[E]$. For $\tau \in \mathcal{C}$ we let

$$
\delta_{\tau}^{\prime}=\text { the maximal } \delta \text { such that } T \uparrow \tau \text { has no cofinal branch in } J_{\delta}^{E} \text {. }
$$

By the above proposition, $\delta_{\tau}^{\prime} \geq \delta_{\tau}$. We would like to pick $\mathbf{L}[E] \| \delta_{\tau}^{\prime}$ as our canonical structure, but the fact that $\tau$ may be collapsed inside $\mathbf{L}[E] \| \delta_{\tau}^{\prime}$ or even definably collapsed over $\mathbf{L}[E] \| \delta_{\tau}^{\prime}$ does not allow to make this choice for each $\tau \in \mathcal{C}$. If a cofinal branch of $T \uparrow \tau$ is introduced later or at the same time when $\tau$ is singularized, $\tau$ will be treated the same way as in the construction of a global square sequence. This motivates our choice of the canonical structure. We define

- $\quad \mathcal{C}^{0}=$ the set of all $\tau \in \mathcal{C}$ such that $\tau$ is singular in $J_{\delta_{\tau}^{\prime}+1}^{E}$.

- $\mathrm{e}^{1}=\mathrm{e}-\mathrm{e}^{0}$.

and

- $N_{\tau}=$ the singularizing level of $\mathbf{L}[E]$ for $\tau$ if $\tau \in \mathcal{C}^{0}$.

- $N_{\tau}=\mathbf{L}[E] \| \delta_{\tau}^{\prime}=\left\langle J_{\delta_{\tau}^{\prime}}^{E}, E_{\omega \delta_{\tau}^{\prime}}\right\rangle$ if $\tau \in \mathcal{C}^{1}$.

Notice that even if $\tau \in \mathcal{C}^{0}$ we have $\operatorname{ht}\left(N_{\tau}\right) \geq \delta_{\tau}$, so $T\left\lceil\tau \in N_{\tau}\right.$ for all $\tau \in \mathcal{C}$.

We first define the sets $C_{\tau}$ witnessing Theorem 0.1 for $\tau \in \lim (\mathcal{C})$. We will treat the cases $\tau \in \mathcal{C}^{i}, i=0,1$ separately and show that the two constructions do not interfere. We begin with $\mathcal{C}^{0}$, as here we can use the global square sequence of [8].

Let $\left\langle C_{\tau}^{\prime} \mid \tau \in \mathcal{S} \cap \kappa\right\rangle$ denote the global square sequence from [8] $]^{2}$ where $\mathcal{S}$ is the class of all singular cardinals. So each $C_{\tau}^{\prime}$ is a closed subset of $\tau$ that is unbounded whenever $\tau$ has uncountable cofinality, the sequence of sets $C_{\tau}^{\prime}$ is fully coherent and $\operatorname{otp}\left(C_{\tau}^{\prime}\right)<\tau$ for each $\tau \in \mathcal{S}$. The class $\mathcal{S}$ is divided into two disjoint classes $\mathcal{S}^{0}$ and $\mathcal{S}^{1}$ and the sets $C_{\tau}^{\prime}$ satisfy the inclusions $C_{\tau}^{\prime} \subseteq \mathcal{S}^{i}$ whenever $\tau \in \mathcal{S}^{i}$ for $i=0,1$. We first make the following observation.

Lemma 1.3 If $\tau \in \lim (\mathcal{C}) \cap \mathcal{C}^{0}$ and $\operatorname{cf}(\tau)>\omega$ then there is some $\gamma<\tau$ such that $C_{\tau}^{\prime} \cap \mathcal{C}-\gamma \subseteq \mathrm{e}^{0}$.

\footnotetext{
2 This is the sequence which is denoted by $\left\langle C_{\tau} \mid \tau \in \mathcal{S}\right\rangle$ in [8]; here we write $C_{\tau}^{\prime}$ instead of $C_{\tau}$, as $C_{\tau}$ will be the final sequence produced in this paper.
} 
Proof Obviously, $C_{\tau}^{\prime} \cap \mathcal{C}$ is unbounded in $\tau$. As $\tau \in \mathcal{C}^{0}$, the canonical structure $N_{\tau}$ is the singularizing $\mathbf{L}[E]$-level for $\tau$. Let $\bar{\tau} \in C_{\tau}^{\prime}$ and $\bar{N}$ be the singularizing $\mathbf{L}[E]$ level for $\bar{\tau}$. By the construction in [8], there is a $\Sigma_{0}$-preserving map $\sigma_{\bar{\tau}, \tau}$ such that $\sigma_{\bar{\tau}, \tau}: \bar{N} \rightarrow N_{\tau}$ if $\tau \in \mathcal{S}^{0}$ and $\sigma_{\bar{\tau}, \tau}: \bar{M} \rightarrow M_{\tau}$ if $\tau \in \mathcal{S}^{1}$; here $\bar{M}$ and $M_{\tau}$ are the canonical protomice assigned to $\bar{\tau}$ and $\tau$. In our situation we have $T \uparrow \tau \in N_{\tau}$, as $\tau \in \mathcal{C}$. First assume $\tau \in \mathcal{S}^{0}$. If $\bar{\tau} \in C_{\tau}^{\prime} \cap \mathcal{C}$ is large enough that $T \uparrow \tau \in \operatorname{rng}\left(\sigma_{\bar{\tau}, \tau}\right)$ then $T \uparrow \bar{\tau} \in \bar{N}, \sigma_{\bar{\tau}, \tau}(T \uparrow \bar{\tau})=T \uparrow \tau$ and $T \uparrow \bar{\tau}$ has no cofinal branch in $N_{\bar{\tau}}$. These conclusions are consequences of the $\Sigma_{0}$-elementarity of the map; the former two follow by an argument similar to that in proof of Lemma 1.2 and the latter one follows from the fact that the nonexistence of a cofinal branch can be expressed as a $\Pi_{1}$-statement, so it is preserved backward under $\sigma_{\bar{\tau}, \tau}$. Hence that $T \uparrow \bar{\tau}$ has no cofinal branch in the singularizing structure for $\bar{\tau}$, and consequently $N_{\bar{\tau}}=\bar{N}$. Now assume $\tau \in \mathcal{S}^{1}$. The conclusion then follows from the fact that $M_{\tau}$ and $N_{\tau}$ compute the cardinal successor of $\tau$ the same way and they agree below this common successor, and the same is true of the structures $\bar{M}$ and $\bar{N}$ and cardinal $\bar{\tau}$ to which they are assigned. The same argument as above can be then used with the map $\sigma_{\bar{\tau}, \tau}$ which is now a map between two protomice. As before we conclude that $\bar{N}=N_{\bar{\tau}}$. It follows that $\bar{\tau} \in \mathrm{C}^{0}$ and the same conclusion can be made for any $\tau^{\prime}$ such that $\bar{\tau} \leq \tau^{\prime}<\tau$, so it suffices to let $\gamma=\bar{\tau}$.

For $\tau \in \lim (\mathcal{C}) \cap \mathcal{C}^{0}$ we let

$$
\gamma_{\tau}=\text { the least } \gamma \leq \tau \text { such that } C_{\tau}^{\prime} \cap \mathcal{C}-\gamma \subseteq \mathcal{C}^{0}
$$

and define $C_{\tau}^{*}$ as follows.

- If $C_{\tau}^{\prime} \cap \mathcal{C}-\gamma_{\tau}$ is unbounded in $\tau$ we let $C_{\tau}^{*}=C_{\tau}^{\prime} \cap \mathcal{C}-\gamma_{\tau}$;

- otherwise $C_{\tau}^{*}$ is the $<_{E}$-least sequence of order type $\omega$ converging to $\tau$.

Lemma 1.3 together with the properties of the sets $C_{\tau}^{\prime}$ guarantee that this definition makes sense, each $C_{\tau}^{*}$ is a closed unbounded subset of $\tau$ and $C_{\bar{\tau}}^{*}=C_{\tau}^{*} \cap \bar{\tau}$ whenever $\bar{\tau} \in \lim \left(C_{\tau}^{*}\right)$. Thus, for $\tau \in \lim (\mathcal{C}) \cap \varrho^{0},(\mathrm{a})$ and (b) in Theorem 0.1 hold with $C_{\tau}^{*}$ in place of $C_{\tau}$.

We next define sets $C_{\tau}^{*}$ for $\tau \in \lim (\mathcal{C}) \cap \mathcal{C}^{1}$. The definition of the sets $C_{\tau}^{*}$ is based on the following observation, which is a direct consequence of the fact that $\tau \in \mathcal{C}^{1}$. Recall that for an acceptable structure $N$, the standard parameter is denoted by $p_{N}$.

$$
\tau \cap \tilde{h}_{N_{\tau}}^{n+1}\left(\alpha \cup\left\{p_{N_{\tau}}\right\}\right) \text { is bounded in } \tau \text { whenever } \alpha<\tau \text { and } n \in \omega \text {. }
$$

The sets $C_{\tau}^{*}$ are defined as follows.

- $C_{\tau}^{*}$ is the set of all $\bar{\tau} \in \tau \cap \mathcal{C}^{1}$ satisfying: $N_{\bar{\tau}}$ is a premouse of the same type as $N_{\tau}$ and there is a $\Sigma^{*}$-preserving embedding $\sigma_{\bar{\tau}, \tau}$ such that:

(i) $\bar{\tau}=\operatorname{cr}(\tau)$ and $\sigma_{\bar{\tau}, \tau}(\bar{\tau})=\tau$.

(ii) $\sigma_{\bar{\tau}, \tau}\left(p_{N_{\bar{\tau}}}\right)=p_{N_{\tau}}$.

(iii) $\sigma_{\bar{\tau}, \tau}(T \uparrow \bar{\tau})=T \uparrow \tau$. 
Clause (iii) in the above definition is superfluous: By the construction of the set $\mathcal{C}$, if $\tau \in \mathcal{C}$ then $J_{\delta_{\tau}}^{E}$ satisfies the statement " $T \uparrow \tau$ is the $<_{E}$-least $\tau$-tree on $\tau$ without a cofinal branch". Moreover, $J_{\delta_{\tau}}^{E}$ is a $\mathrm{ZFC}^{-}$-model since it can be elementarily embedded into $J_{\kappa^{+}}^{E}$, so $N_{\tau}$ is a proper extension of $J_{\delta_{\tau}}^{E}$. So the map $\sigma_{\bar{\tau}, \tau}$ is sufficiently elementary to satisfy (iii) even if we did not require it explicitly. We include it as a part of the definition in order to simplify the matters. Clearly, the map $\sigma_{\bar{\tau}, \tau}$ is the unique $\Sigma^{*}$-preserving map $\sigma: N_{\bar{\tau}} \rightarrow N_{\tau}$ satisfying (i) and (ii).

Lemma 1.4 If $\tau \in \lim (\mathcal{C}) \cap \mathcal{C}^{1}$ and $\operatorname{cf}(\tau)>\omega$ then $C_{\tau}^{*}$ is unbounded in $\tau$.

Proof Given some $\tau^{*}<\tau$ we find $\bar{\tau} \in C_{\tau}^{*}$ such that $\tau^{*} \leq \bar{\tau}$. As is typical for constructions of square sequences, we will look for the right kind of hulls. Recall that if $N$ is an acceptable structure, $p \in N$ is a finite set of ordinals and $v$ is an ordinal then $W_{N}^{v, p}$ is the standard witness for $p$ with respect to the ordinal $v$ and structure $N$. Let

- $n \in \omega$ be such that $\omega \varrho_{N_{\tau}}^{n+1} \leq \tau<\omega \varrho_{N_{\tau}}^{n}$;

Such an $n$ exists, as there is a cofinal branch through $T \uparrow \tau$ in $J_{\delta_{\tau}^{\prime}+1}^{E}-J_{\delta_{\tau}^{\prime}}^{E}$ and such a branch, being a subset of $\tau$, is $\Sigma^{*}$-definable over $J_{\delta_{\tau}^{\prime}+1}^{E} \| \delta_{\tau}^{\prime}=N_{\tau}$. Let $x \in[\tau]^{<\omega}$ be such that $T\left\lceil\tau \in \tilde{h}_{N_{\tau}}^{n+1}\left(x \cup\left\{p_{N_{\tau}}\right\}\right), W_{N_{\tau}}^{\nu, p_{\tau}} \in \tilde{h}_{N_{\tau}}^{n+1}\left(x \cup\left\{p_{N_{\tau}}\right\}\right)\right.$ whenever $v \in p_{N_{\tau}}$, and some cofinal branch through $T \uparrow \tau$ is $\Sigma_{1}^{(n)}\left(N_{\tau}\right)$-definable from $x$ and $p_{N_{\tau}}$. Such an $x$ exists, as these tasks require only a finite amount of information. Define a sequence $\left\langle\tau_{k}, X_{k} \mid k \in \omega\right\rangle$ of ordinals below $\tau$ and hulls as follows.

$$
\begin{aligned}
\tau_{0} & =\max \left(x \cup\left\{\tau^{*}\right\}\right)+1 \\
X_{k} & =\bigcup_{\ell \in \omega} \tilde{h}_{N_{\tau}}^{\ell+1}\left(\tau_{k} \cup\left\{p_{N_{\tau}}\right\}\right) \\
\tau_{k+1}^{\prime} & =\sup \left(\tau \cap X_{k}\right) \\
\tau_{k+1} & =\min \left(\mathcal{C}-\tau_{k+1}^{\prime}\right)+1
\end{aligned}
$$

By (2), each $\tau \cap \tilde{h}_{N_{\tau}}^{\ell+1}\left(\tau_{\kappa} \cup\left\{p_{N_{\tau}}\right\}\right)$ is smaller than $\tau$, granting that $\tau_{k}<\tau$. Since $\tau$ has uncountable cofinality, also $\tau_{k+1}<\tau$, which enables us to run induction on $k$ and then conclude that also $\bar{\tau}=\sup \left(\left\{\tau_{k} \mid k \in \omega\right\}\right)$ is below $\tau$. Letting $X=\bigcup_{k \in \omega} X_{k}$ we have $\tau \cap X=\bar{\tau}$. In the following we show that $\bar{\tau} \in C_{\tau}^{*}$.

Notice first that since the ordinals $\tau_{k}$ are strictly increasing and each interval $\left(\tau_{k}, \tau_{k+1}\right)$ has nonempty intersection with $\mathcal{C}$, the supremum $\bar{\tau}$ is a limit point of $\mathcal{C}$, so $\bar{\tau} \in \mathcal{C}$. We next observe:

$$
X=\tilde{h}_{N_{\tau}}^{n+1}\left(\bar{\tau} \cup\left\{p_{N_{\tau}}\right\}\right) .
$$

By construction, each $z \in X$ is of the form $\tilde{h}_{N_{\tau}}^{\ell+1}\left(i,\left\langle\bar{z}, p_{N_{\tau}}\right\rangle\right)$ for some $\ell \geq n, i \in \omega$ and $\bar{z} \in[\bar{\tau}]^{<\omega}$. Assume $\ell>n$. The function $\tilde{h}_{N_{\tau}}^{\ell+1}\left(u,\left\langle v, p_{N_{\tau}}\right\rangle\right)$ can be expressed as a composition $\tilde{h}_{N_{\tau}}^{n+1}\left((u)_{0}^{2},\left\langle h\left((u)_{1}^{2}, v\right), p_{N_{\tau}}\right\rangle\right)$ where $h: \omega \times J_{\tau}^{E} \rightarrow J_{\tau}^{E}$ is a partial $\operatorname{good} \Sigma_{1}^{(\ell)}$-function; see [7], Sect. 1.8 for details. Here $(u)_{i}^{2}$ is the $i$-th component of $u$ 
under the Gödel pairing function for $i=0$, 1. If $\bar{z} \in\left[\tau_{k}\right]^{<\omega}$ then $h\left((u)_{1}^{2}, \bar{z}\right) \in J_{\tau_{k}^{\prime}}^{E}$, and since there is a uniformly $\Sigma_{1}$-definable surjection of $\left[\tau_{k}^{\prime}\right]^{<\omega}$ onto $J_{\tau_{k}^{\prime}}^{E}$ we can replace the above value of $h$ with some finite $z^{\prime} \in\left[\tau_{k}^{\prime}\right]^{<\omega}$. So there is some $j<\omega$ such that $z=\tilde{h}_{N_{\tau}}^{n+1}\left(j,\left\langle z^{\prime}, p_{N_{\tau}}\right\rangle\right)$. This proves (3).

Let $\bar{N}$ be the transitive collapse of $X$ and $\sigma: \bar{N} \rightarrow N_{\tau}$ be the inverse to the Mostowski collapsing isomorphism. Then $\bar{\tau}=\operatorname{cr}(\sigma)$ and $\sigma(\bar{\tau})=\tau$. Moreover, it follows from (3) and the construction of $X$ that

$$
X=\tilde{h}_{N_{\tau}}^{\ell+1}\left(\bar{\tau} \cup\left\{p_{N_{\tau}}\right\}\right) \quad \text { whenever } \ell \geq n,
$$

so the map $\sigma$ is $\Sigma_{1}^{(\ell)}$-preserving for all such $\ell$ hence $\Sigma^{*}$-preserving. Here (3) directly yields the inclusion " $\subseteq$ "; from the construction of $X$ we obtain the converse. As $x, T \nmid \tau \in X$, we have $x, T\lceil\bar{\tau} \in \bar{N}$ and $\sigma(x, T \nmid \bar{\tau})=(x, T\lceil\tau)$. For $x$ this is immediate, for $T\lceil\tau$ this follows from the fact that $T\lceil\tau \in \operatorname{rng}(\sigma)$ by an argument similar to that in the proof of Lemma 1.2. Since $p_{\tau} \in X$ we have some $\bar{p} \in \bar{N}$ such that $\sigma(\bar{p})=p_{\tau}$. From (3) we obtain $\bar{N}=\tilde{h}_{\bar{N}}^{n+1}(\bar{\tau} \cup\{\bar{p}\})$ which implies that $\omega \varrho_{\bar{N}}^{n+1} \leq \bar{\tau}$ and $\bar{p} \in R_{\bar{N}}^{n+1} \cdot{ }^{3}$ By construction $\bar{\tau}$ is a limit cardinal in $\mathbf{L}[E]$, so actually $\omega \varrho_{\bar{N}}^{\omega}=\omega \varrho_{\bar{N}}^{n+1}=\tau$. The structure $\bar{N}$ is a premouse of the same type as $N$; this follows from the $\Sigma^{*}$-elementarity of $\sigma$.

An application of the Condensation Lemma to the map $\sigma: \bar{N} \rightarrow N_{\tau}$ then yields that $\bar{N}$ is a solid premouse. The choice of the set $x$ at the beginning of the construction guarantees that for each $v \in p_{\tau}$ the standard witness $W_{N_{\tau}}^{\mathcal{v}, p_{\tau}}$ is an element of $X$, so its preimage $Q_{\nu}$ under $\sigma$ is a generalized witness for $\bar{v}=\sigma^{-1}(\nu) \in \bar{p}$ with respect to $M$ and $\bar{p} .{ }^{4}$ So $\bar{p}=p_{\bar{N}}$ and $\bar{N}$ is sound above $\bar{\tau}$ by [7], Lemma 1.12.5. One more application of the Condensation Lemma to the map $\sigma: \bar{N} \rightarrow N$ then gives us the following options: (a) $\bar{N}=\operatorname{core}(N)$, (b) $\bar{N}$ is a proper initial segment of $N$, (c) $\bar{N}$ is an ultrapower of an initial segment $N^{\prime}$ of $N$ with critical point equal to the cardinal predecessor of $\bar{\tau}$ in $N^{\prime}$ and (d) $\bar{N}$ is a proper initial segment of $\operatorname{Ult}\left(N, E_{\bar{\tau}}\right)$. Here option (a) is impossible as $\bar{N}$ and $N$ have different ultimate projecta and options (c) and (d) are impossible as $\bar{\tau}$ is a limit cardinal in $\bar{N}$. Thus, $\bar{N}$ is a proper initial segment of $N_{\tau}$ and thereby an initial segment of $\mathbf{L}[E]$.

So far we have obtained an initial segment $\bar{N}$ of $\mathbf{L}[E]$ and a $\Sigma^{*}$-preserving map $\sigma: \bar{N} \rightarrow N_{\tau}$ such that $\tau^{*}<\bar{\tau}=\operatorname{cr}(\sigma)$ is inaccessible in $\bar{N}$ and $\sigma\left(\bar{\tau}, p_{\bar{N}}, T\lceil\bar{\tau})=\right.$ $\left(\tau, p_{\tau}, T\lceil\tau)\right.$. Obviously, $T\lceil\bar{\tau}$ has no cofinal branch in $\bar{N}$, as $T\lceil\tau$ has no cofinal branch in $N_{\tau}$ and $\sigma$ is sufficiently elementary. In order to verify that $\bar{\tau} \in C_{\tau}^{*}$ we have to verify that $\bar{\tau} \in \mathcal{C}^{1}$ which amounts to showing that $\bar{N}=N_{\bar{\tau}}$. This is equivalent to saying that $\bar{\tau}$ is regular in $J_{\beta+1}^{E}$ and $T \nmid \bar{\tau}$ has a cofinal branch in $J_{\beta+1}^{E}$ where $\beta=\operatorname{ht}\left(N_{\bar{\tau}}\right)$. The former follows immediately from the construction of $X$, as the $\Sigma^{*}$ elementarity of $\sigma$ implies that $\tilde{h}_{\bar{N}}^{\ell+1}\left(\tau_{k} \cup\left\{p_{\bar{N}}\right\}\right)$ is bounded in $\bar{\tau}$ for all $k, \ell \in \omega$. As any function $f: \bar{\tau} \rightarrow \bar{\tau}$ that is an element of $J_{\beta+1}^{E}$ is definable over $\bar{N}$ and therefore

\footnotetext{
${ }^{3}$ See [7], Sect. 1.5

${ }^{4}$ See ([7]), Sect. 1.12 or ([8])
} 
can be expressed in the form $\tilde{h}_{\bar{N}}^{\ell+1}\left(-\cup\left\{q \cup p_{\bar{N}}\right\}\right)$ for some $\ell \in \omega$ and $q \in[\bar{\tau}]^{<\omega}$, such function cannot singularize $\bar{\tau}$.

To see that $J_{\beta+1}^{E}$ contains a cofinal branch through $T \uparrow \bar{\tau}$ it suffices to show that such a branch is $\Sigma^{*}$-definable over $\bar{N}$. Let $b \in J_{\delta_{\tau}^{\prime}+1}^{E}$ be a cofinal branch through $T \uparrow \tau$. Similarly as with $f$ above, it follows that $b$ is $\Sigma_{1}^{(\ell)}\left(N_{\tau}\right)$-definable from $p_{N_{\tau}}$ and some $q \in[\tau]^{<\omega}$ for some $\ell \in \omega$. Let $\varphi(u, v)$ be a $\Sigma_{1}^{(\ell)}$-formula that defines $b$, that is, for each $\xi<\tau$ we have

$$
\xi \in b \Longleftrightarrow N_{\tau} \models \varphi\left(\xi, q \cup p_{N_{\tau}}\right)
$$

We may without loss of generality assume that $\ell \geq n$. We first observe that $q$ can be taken from $[\bar{\tau}]^{<\omega}$. This is the case, as the statement "the set of all $\xi<\tau$ satisfying $N_{\tau} \models \varphi\left(\xi, q \cup p_{N_{\tau}}\right)$ determines a cofinal branch through $T \uparrow \tau$ " can be expressed in a $\Pi_{1}^{(\ell+2)}$-manner, namely as the conjunction of

$$
\begin{aligned}
& \left(\forall \xi^{\ell+1}, \zeta^{\ell+1}\right)\left[\left(\varphi\left(\xi^{\ell+1}, q \cup p_{N_{\tau}}\right) \& \varphi\left(\zeta^{\ell+1}, q \cup p_{N_{\tau}}\right)\right)\right. \\
& \left.\longrightarrow\left(\xi^{\ell+1}<_{T} \zeta^{\ell+1} \vee \zeta^{\ell+1}<_{T} \xi^{\ell+1}\right)\right]
\end{aligned}
$$

and

$$
\left(\forall \xi^{\ell+2}\right)\left(\exists \zeta^{\ell+1}\right)\left(\zeta^{\ell+1}>\xi^{\ell+2} \& \varphi\left(\zeta^{\ell+2}, q \cup p_{N_{\tau}}\right)\right)
$$

The former expresses that $b$ determines a branch through $T \uparrow \tau$ and the latter expresses that the branch is cofinal. Recall that $T \uparrow \tau$ consists of ordinals smaller than $\tau$ and we chose $n$ so that $\omega \varrho_{N_{\tau}}^{n} \leq \tau$. Since $\tau$ is an $\mathbf{L}[E]$-cardinal by Lemma 1.2, we actually have $\omega \varrho_{N_{\tau}}^{n}=\tau=\omega \varrho_{N_{\tau}}^{\omega}$. This allows us to use variables $\xi^{\ell+1}$ and $\xi^{\ell+1}$ in the formulae above. The conjunction of these formulae is a statement about $q$ and $p_{N_{\tau}}$; denote it by $\psi\left(q, p_{N_{\tau}}\right)$. As $q$ witnesses that $N_{\tau} \models\left(\exists z^{\ell+3}\right) \psi\left(z, p_{N_{\tau}}\right)$ and $X$ is closed under good $\Sigma^{*}$-functions (again, the variable $z^{\ell+3}$ can be used for the same reason as explained above), there also must be a witness $\bar{q} \in X$. Then $\bar{q} \in[\bar{\tau}]^{<\omega}$ and $\bar{N} \models \psi\left(\bar{q}, p_{\bar{N}}\right)$. It follows that $\left\{\xi<\bar{\tau} \mid \bar{N} \models \varphi\left(\xi, \bar{q} \cup p_{\bar{N}}\right)\right\}$ determines a cofinal branch through $T\lceil\bar{\tau}$. Such a branch is $\Sigma_{1}^{(\ell)}$-definable over $\bar{N}$ in parameters. This completes the proof of the fact that $\bar{\tau} \in \mathcal{C}^{1}$ and thereby the proof of the lemma.

Lemma 1.5 If $\tau \in \lim (\mathcal{C}) \cap \mathcal{C}^{1}$ then $C_{\tau}^{*}$ is closed.

Proof Let $\bar{\tau}$ be a limit point of $C_{\tau}^{*}$. We show that $\bar{\tau} \in C_{\tau}^{*}$. As in the previous lemma, let

- $\quad n$ be such that $\omega \varrho_{N_{\tau}}^{n+1} \leq \tau<\omega \varrho_{N_{\tau}}^{n}$.

We first observe that if $\tau^{*} \in C_{\tau}^{*}$ then $\omega \varrho_{N_{\tau^{*}}}^{n+1} \leq \bar{\tau}<\omega \varrho_{N_{\tau^{*}}}^{n}$. The inequality on the right follows from the fact that $N_{\tau}$ satisfies the $\Sigma_{1}^{(n)}$-statement $\left(\exists \xi^{n}\right)\left(\tau<\xi^{n}\right)$ and this statement is preserved under $\sigma$. The inequality on the left follows from the fact 
that $\tilde{h}_{N_{\tau}}^{n+1}\left(\tau \cup\left\{p_{N_{\tau}}\right\}\right)=N_{\tau}$ and $\sigma$ preserves $\Sigma_{1}^{(n)}$-statements. Consider the diagram $\left\langle N_{\tau^{*}}, \sigma_{\tau^{*}, \tau^{\prime}} \mid \tau^{*} \leq \tau^{\prime} \in \bar{\tau} \cap C_{\tau}^{*}\right\rangle$; let $\left\langle\bar{N}, \sigma_{\tau^{*}} \mid \tau^{*} \in \bar{\tau} \in C_{\tau}^{*}\right\rangle$ be its direct limit with the direct limit maps $\sigma_{\tau^{*}}: N_{\tau^{*}} \rightarrow \bar{N}$ and let $\bar{\sigma}: \bar{N} \rightarrow N_{\tau}$ be the canonical embedding of the direct limit $\bar{N}$ into $N_{\tau}$ satisfying $\bar{\sigma} \circ \sigma_{\tau^{*}}=\sigma_{\tau^{*}, \tau}$. Standard considerations yield that $\bar{N}$ can be viewed as a premouse of the same type as $N_{\tau}$ and all $N_{\tau^{*}}$, all maps $\sigma_{\tau^{*}}$ and $\bar{\sigma}$ are $\Sigma^{*}$-preserving and $\omega \varrho_{\bar{N}}^{n+1} \leq \bar{\tau}<\omega \varrho_{\bar{N}}^{n}$. If $\bar{p}$ is the common value of $\sigma_{\tau^{*}}\left(p_{N_{\tau^{*}}}\right)$ then obviously $\bar{\sigma}(\bar{p})=p_{N_{\tau}}$. By the properties of $n$ recorded above, $\tilde{h}_{N_{\tau}}^{n+1}\left(\tau \cup\left\{p_{N_{\tau}}\right\}\right)=N_{\tau}$ so $\tilde{h}_{\bar{N}}^{n+1}(\bar{\tau} \cup\{\bar{p}\})=\bar{N}$, as follows from preservation properties of $\bar{\sigma}$. An application of the Condensation Lemma to the map $\bar{\sigma}: \bar{N} \rightarrow N_{\tau}$ yields that $\bar{N}$ is solid. Since each $N_{\tau^{*}}$, being a proper initial segment of $\mathbf{L}[E]$, is sound, for each $v \in p_{N_{\tau^{*}}}$ the standard witness $W_{N_{\tau^{*}}}^{v, p_{\tau^{*}}}$ is an element of $N_{\tau^{*}}$ and its image under $\sigma_{\tau^{*}}$ is a generalized witness for $\sigma_{\tau^{*}}(\nu)$ with respect to $\bar{N}$ and $\bar{p}$, as $\sigma_{\tau^{*}}$ is sufficiently elementary. This way we conclude that for each element of $\bar{p}$ there is in $\bar{N}$ a generalized witness with respect to $\bar{N}$ and $\bar{p}$, and exactly as in the proof of Lemma 1.4 then conclude that $\bar{N}$ is sound and $\bar{p}=p_{\bar{N}}$. One more application of the Condensation Lemma then yields, exactly as in Lemma 1.4 that $\bar{N}$ is an initial segment of $N_{\tau}$. Obviously $\bar{\tau}=\operatorname{cr}(\sigma), \sigma\left(\bar{\tau}, p_{\bar{N}}\right)=\tau, p_{N_{\tau}}$, the cardinal $\bar{\tau}$ is inaccessible in $\bar{N}$ and $\bar{N}$, being a limit point of $C_{\tau}^{*}$, is a limit point of $\mathcal{C}$ hence $\bar{\tau} \in \mathcal{C}$. It remains to prove that $\bar{\tau} \in \mathcal{C}^{1}$. As $\sigma$ is $\Sigma^{*}$-preserving, this follows exactly as in the proof of Lemma $1.4 .^{5}$

Lemma 1.6 If $\tau \in \lim (\bigodot) \cap \complement^{1}$ and $\bar{\tau} \in \lim \left(C_{\tau}^{*}\right)$ then $\bar{\tau} \in \lim (\bigodot) \cap \bigodot^{1}$ and $C_{\bar{\tau}}^{*}=$ $C_{\tau}^{*} \cap \bar{\tau}$.

Proof Since $\tau \in \lim (\mathcal{C}) \cap \mathcal{C}^{1}$, the condition $\bar{\tau} \in \lim \left(C_{\tau}^{*}\right)$ implies $\bar{\tau} \in \lim (\mathcal{C}) \cap \mathcal{C}^{1}$, so $C_{\tau}^{*}$ and $C_{\bar{\tau}}^{*}$ are defined in the same way. If $\tau^{*} \in C_{\bar{\tau}}$ then $\tau^{*} \in \mathrm{C}^{1}$ and we have the map $\sigma_{\tau^{*}, \bar{\tau}}: N_{\tau^{*}} \rightarrow N_{\bar{\tau}}$ witnessing the membership of $\tau^{*}$ to $C_{\bar{\tau}}^{*}$. But then $\sigma_{\bar{\tau}, \tau} \circ \sigma_{\tau^{*}, \bar{\tau}}$ : $N_{\tau^{*}} \rightarrow N_{\tau}$ witnesses the membership of $\tau^{*}$ to $C_{\tau}$. Conversely, if $\tau^{*} \in C_{\tau}^{*} \cap \bar{\tau}$ then $\tau^{*} \in \mathcal{C}^{1}$ and there is a map $\sigma_{\tau^{*}, \tau}$ witnessing the membership of $\tau^{*}$ to $C_{\tau}$. Since both $\sigma_{\tau^{*}, \tau}$ and $\sigma_{\bar{\tau}, \tau}$ are $\Sigma^{*}$-preserving and $\tau^{*}<\bar{\tau}$ we have

$$
\operatorname{rng}\left(\sigma_{\tau^{*}, \tau}\right)=\bigcup_{\ell \in \omega} \tilde{h}_{N_{\tau}}^{\ell+1}\left(\tau^{*} \cup\left\{p_{N_{\tau}}\right\}\right) \subseteq \bigcup_{\ell \in \omega} \tilde{h}_{N_{\tau}}^{\ell+1}\left(\bar{\tau} \cup\left\{p_{N_{\tau}}\right\}\right)=\operatorname{rng}\left(\sigma_{\bar{\tau}, \tau}\right),
$$

so $\left(\sigma_{\bar{\tau}, \tau}\right)^{-1} \circ \sigma_{\tau^{*}, \tau}: N_{\tau^{*}} \rightarrow N_{\bar{\tau}}$ witnesses the membership of $\tau^{*}$ to $C_{\bar{\tau}}^{*}$.

So far we have constructed sets $C_{\tau}^{*}$ for $\tau \in \lim (\mathcal{C})$ such that (a) and (b) in Theorem 0.1 hold with $C_{\tau}^{*}$ in place of $C_{\tau}$. Given a stationary set $A \subseteq \kappa$, we find a stationary $A^{\prime} \subseteq A$ and refine $C_{\tau}^{*}$ into $C_{\tau}$ that will satisfy all conclusions of the Theorem. We let

- $\quad A^{\prime}=$ the set of all $\tau \in \mathcal{C}$ for which there are an $\mathbf{L}[E]$-level $P=J_{\beta}^{E}$ and a parameter $a \in P$ such that:

\footnotetext{
5 Alternatively, one can consider a definition of a cofinal branch of $T \uparrow \tau^{*}$ over $N_{\tau^{*}}$ from parameters $p_{\tau^{*}}$ and $q \in\left[\tau^{*}\right]^{<\omega}$ for some/any $\tau^{*} \in C_{\tau}^{*} \cap \bar{\tau}$ and show that the same definition over $\bar{N}$ defines a cofinal branch through $T \uparrow \bar{\tau}$ from $p_{\bar{N}}$ and $q$. This works, as $\sigma_{\tau^{*}}$ is $\Sigma^{*}$-preserving.
} 
(a) $P \models \mathrm{ZFC}^{-}, \tau$ is the largest cardinal in $P$, is inaccessible in $P$ and $T \uparrow \tau$ has no cofinal branch in $P$.

(b) For every $X \prec P$ satisfying $X \cap \tau \in \tau$ and $p \in X$ we have $X \cap \tau \notin A$.

The same proof as that of [8, Theorem 3.21] shows that the set $A^{\prime}$ is stationary in $\kappa$. Notice that the only difference between $A^{\prime}$ in this paper and $A^{\prime}$ in [8] is the additional requirement in (a) above that $T \uparrow \tau$ has no cofinal branch in $P$ and the restriction of the set $A^{\prime}$ to elements of the closed unbounded set $\mathrm{C}$.

Lemma 1.7 Let $\tau \in \lim (\mathcal{C})$. If $\bar{\tau} \in \lim \left(C_{\tau}^{*}\right) \cap A^{\prime}$ then there is some $\tau^{*} \in C_{\tau}^{*} \cap \bar{\tau}$ such that $A$ is disjoint with $C_{\tau}^{*} \cap\left(\tau^{*}, \bar{\tau}\right)$.

Proof For $\tau \in \mathcal{C}^{0}$ this was proved in [8], Lemma 3.22. For $\tau \in \mathcal{C}^{1}$ the same argument goes through. If there is a pair $(P, a) \in N_{\bar{\tau}}$ witnessing the membership of $\bar{\tau}$ to $A^{\prime}$ the argument can be literally repeated: Given $\tau^{\prime} \in C_{\tau}^{*} \cap \bar{\tau}$ large enough that $(P, a)$ is in the range of $\sigma_{\tau^{\prime}, \bar{\tau}}$, let $P^{\prime} \in N_{\tau^{\prime}}$ be such that $\sigma_{\tau^{\prime}, \bar{\tau}}\left(P^{\prime}\right)=P$; then $X=\sigma_{\tau^{\prime}, \bar{\tau}}\left[P^{\prime}\right] \prec P$ and $a \in X$, so $\tau^{\prime}=X \cap \bar{\tau} \notin A$. In the remaining case we conclude that $P=J_{\delta_{\bar{\tau}}^{\prime}}^{E}$ where recall that $\delta_{\bar{\tau}}^{\prime}=\operatorname{ht}\left(N_{\bar{\tau}}\right)$. This is the case, as $T \uparrow \bar{\tau}$ has a cofinal branch in $J_{\delta_{\bar{\tau}+1}^{\prime}}^{E}$. As $P \models \mathrm{ZFC}^{-}$and $N_{\bar{\tau}}$ projects to $\bar{\tau}$, necessarily $E_{\text {top }}^{N_{\bar{\tau}}}$ is an extender with $\lambda\left(E_{\text {top }}^{N_{\bar{\tau}}}\right)=\bar{\tau}$. Since $\sigma_{\tau^{\prime}, \bar{\tau}}$ is $\Sigma^{*}$-preserving, $E_{\text {top }}^{N_{\tau^{\prime}}}$ is an extender with $\lambda\left(E_{\text {top }}^{N_{\tau^{\prime}}}\right)=\tau^{\prime}$ and the two extenders have the same critical point $\mu<\tau^{\prime}$. Moreover, since both $\tau^{\prime}$ and $\bar{\tau}$ are limit cardinals, both $N_{\tau^{\prime}}$ and $N_{\bar{\tau}}$ compute the cardinal successor of $\mu$ the same way as $\mathbf{L}[E]$; denote this common successor by $\vartheta$. As both these premice are coherent structures, $J_{\delta_{\tau^{\prime}}^{\prime}}^{E}=\operatorname{Ult}\left(J_{\vartheta}^{E}, E_{\text {top }}^{N_{\tau^{\prime}}}\right), J_{\delta_{\bar{\tau}}^{\prime}}^{E}=\operatorname{Ult}\left(J_{\vartheta}^{E}, E_{\text {top }}^{N_{\bar{\tau}}}\right)$ and it follows immediately that $\sigma_{\tau^{\prime}, \bar{\tau}}: \pi^{\prime}(f)(\alpha) \mapsto \bar{\pi}(f)\left(\sigma_{\tau^{\prime}, \bar{\tau}}(\alpha)\right)$ and therefore is fully elementary. Hence if $a \in \operatorname{rng}\left(\sigma_{\tau^{\prime}, \bar{\tau}}\right)$ then $X=\operatorname{rng}\left(\sigma_{\tau^{\prime}, \bar{\tau}}\right) \prec P$. It follows that $\tau^{\prime}=X \cap \bar{\tau} \notin A$.

For $\tau \in \lim (\mathcal{C})$ we can now define sets $C_{\tau}$ as in [8]. We first let

$$
\delta_{\tau}=\text { the least } \delta \leq \tau \text { such that } A \cap C_{\tau}^{*}-\delta=\varnothing
$$

We then let

$$
C_{\tau}=C_{\tau}^{*}-\bigcup\left\{\left(\delta_{\bar{\tau}}, \bar{\tau}\right) \mid \bar{\tau} \in \lim \left(C_{\tau}^{*}\right) \cap A^{\prime}\right\}
$$

Then the sets $C_{\tau}$ are obviously closed. If $A^{\prime} \cap \lim \left(C_{\tau}^{*}\right)$ is bounded in $\tau$ then $C_{\tau}$ is clearly unbounded; otherwise $C_{\tau}$ is unbounded because it follows from its definition that $A^{\prime} \cap \lim \left(C_{\tau}^{*}\right) \subseteq C_{\tau}$. The coherency of the sets $C_{\tau}$ follows from the coherency of the sets $C_{\tau}^{*}$ and the uniformity of the definition of $C_{\tau}$. Finally $\lim \left(C_{\tau}\right) \cap A^{\prime}=\varnothing$, as every element of $A^{\prime}$ is a successor point of $C_{\tau}$.

It remains to define the sets $C_{\tau}$ for $\tau \notin \lim (\mathcal{C})$. Notice that $A^{\prime} \subseteq \mathcal{C}$, which simplifies the matters. The complement of $\lim (\mathcal{C})$ can be written as the union of disjoint open intervals that are bounded in $\kappa$. We assume that these intervals are maximal. Let $(\alpha, \beta)$ be such an interval. Then $\alpha, \beta \in \lim (\mathcal{C})$ by maximality. The set $C_{\beta}$ is defined above, and it has no limit points in the interval $(\alpha, \beta)$. For each $\tau \in(\alpha, \beta)$ we can thus let 
$C_{\tau}=\tau-(\alpha+1)$. Obviously, this definition does not collide with the definition in the case where $\tau \in \lim (\mathcal{C})$ and satisfies (a)-(c) in Theorem 0.1 . This completes the entire construction.

Open Access This article is distributed under the terms of the Creative Commons Attribution Noncommercial License which permits any noncommercial use, distribution, and reproduction in any medium, provided the original author(s) and source are credited.

\section{References}

1. Devlin, K.J.: Constructibility. Springer, New York (1984)

2. Cox, S.: Covering Theorems for the core model, and an application to stationary set reflection. Ann. Pure Appl. Log. 161(1), 66-93 (2009)

3. Jensen, R.B.: The fine structure of the constructible hierarchy. Ann. Math. Log. 4, 229-308 (1972)

4. Jensen, R.B.: A new fine structure for higher core models, handwritten notes, Berlin (1997)

5. Schimmerling, E., Zeman, M.: Square in core models, square in core models. Bull. Symb. Log. 7(3), 305-314 (2001)

6. Schimmerling, E., Zeman, M.: Characterization of $\square_{\kappa}$ in core models. J. Math. Log. 4(1)1,1-72 (2004)

7. Zeman, M.: Inner Models and Large Cardinals, de Gruyter Series in Logic and its Applications 5. De Gruyter, New York (2002)

8. Zeman, M.: Global square sequences in extender models, (submitted), preprint available at http://math. uci.edu/ mzeman/RESEARCH 